\title{
Preface: Mechanical characterization of soft materials
}

Determining the mechanical properties of soft materials (e.g., biological soft tissues, polymeric gels, and soft elastomers) across multiple length scales represents an activity of increasing importance in understanding the deformation behavior of soft materials under various stimuli. Much effort has been made in the past in this field. This theme issue of the Acta Mechanica Sinica contains a number of innovative papers regarding the mechanical characterization of soft materials from five different groups.

X. H. Zhao et al. proposed a method to separate the poroelasticity and viscoelasticity of gels at different time scales by setting the sample lengths to be much larger or smaller than the material lengths of the gels. A theory of ideal linear poroviscoelastic gel was presented and implemented into a finite element model. Both finite element simulations and experiments have been carried out to validate their method in separating the viscoelasticity and poroelasticity of gels at different time scales. M. Oyen and coworkers used spherical indentation to characterize the mechanical properties of hydrogel systems. In particular, the relationships between elastic modulus, viscoelastic ratio, and gel concentration were explored. They showed that there was an increase in modulus with an increase in the total gel concentration; whereas the viscoelastic ratio was less dependent upon total polymer concentration, and more on the particular chemistry of the system. Their study demonstrated that the mechanical characterization of hydrogel systems was essential in understanding cellular behavior on the substrates with different elastic properties. J. Chen et al. measured the mechanical properties of the PDMS substrates using nanoindentation tests. They showed that the instantaneous moduli of the PDMS with different crosslink densities were quite sim- ilar, but the long-term modulus of the PDMS decreased with the decrease of crosslink density. In addition, their results showed that crosslink density had significantly effects on surface adhesion, the PDMS with the lowest crosslink density showed a significantly higher surface adhesion. An important conclusion given by the authors was that the surface adhesion of PDMS could contribute to cell-materials interactions besides the substrate modulus. Determination of the mechanical properties of biofilms is of great importance and represents a challenging issue. N. H. Zhang and co-workers proposed an analytical model to evaluate the mechanical properties of a dsDNA biofilm. The elastic modulus of the DNA biofilm predicted by their model was about 10MPa, in agreement with experimental results. Their study showed that an increment in packing density of the DNA chains or a reduction in salt concentration of the buffer solution could both help increase Young's modulus of the biofilm. RaviChandar et al. studied the time-dependent deformation behavior of pig skins. Dynamic stresses and strains have been measured for various strain rates. Their results should help understand the response of pig skins subjected to a range of loading conditions, including impact and penetration in biomedical applications, which will be published on Vol.30 Iss.2.

The contributions contained in this special issue demonstrate the importance and challenge in the field of mechanical characterization of soft materials, which has been and will continue to be a vital topic in soft materials research for the foreseeable future. It has been the privilege of the guest editors to organize this special issue. We wish to thank all the authors and reviewers for their valuable contributions.

Yan-Ping Cao and Yang-Tse Cheng

\footnotetext{
Y.-P. Cao (四)

Institute of Biomechanics and Biomedical Engineering

Department of Engineering Mechanics

Tsinghua University, 100084 Beijing, China

e-mail: caoyanping@tsinghua.edu.cn

Y.-T. Cheng

Department of Chemical and Materials Engineering,

University of Kentucky, 177 FPAT,

Lexington, KY 40506, USA

e-mail: yang.t.cheng@uky.edu
} 